\title{
Robotic surgery, video-assisted thoracic surgery, and open surgery for early stage lung cancer: comparison of costs and outcomes at a single institute
}

\author{
Pierluigi Novellis ${ }^{1}$, Edoardo Bottoni ${ }^{1}$, Emanuele Voulaz ${ }^{1}$, Umberto Cariboni ${ }^{1}$, Alberto Testori ${ }^{1}$, Luca \\ Bertolaccini $^{2}$, Laura Giordano ${ }^{3}$, Elisa Dieci ${ }^{1}$, Lorenzo Granato ${ }^{4}$, Elena Vanni ${ }^{4,5}$, Marco Montorsi ${ }^{5,6}$, Marco \\ Alloisio $^{1,5}$, Giulia Veronesi ${ }^{1}$ \\ ${ }^{1}$ Division of Thoracic Surgery, Humanitas Clinical and Research Center, Rozzano, Milan, Italy; ${ }^{2}$ Department of Thoracic Surgery, Maggiore \\ Teaching Hospital, Bologna, Italy; ${ }^{3}$ Division of Biostatistics, Department of Oncology, Humanitas Clinical and Research Center, Rozzano, Milan, \\ Italy; ${ }^{4}$ Humanitas Clinical and Research Center, Business Operating Officer, Rozzano, Milan, Italy; ${ }^{5}$ Department of Biomedical Science, Humanitas \\ University, Rozzano, Milan, Italy; ${ }^{6}$ Division of General Surgery, Humanitas Clinical and Research center, Rozzano, Milan, Italy \\ Contributions: (I) Conception and design: G Veronesi, P Novellis; (II) Administrative support: E Vanni, L Granato; (III) Provision of study materials \\ or patients: U Cariboni, A Testori, G Veronesi, M Alloisio; (IV) Collection and assembly of data: E Dieci, P Novellis; (V) Data analysis and \\ interpretation: G Veronesi, P Novellis, L Giordano; (VI) Manuscript writing: All authors; (VII) Final approval of manuscript: All authors. \\ Correspondence to: Pierluigi Novellis. Division of Thoracic Surgery, Humanitas Clinical and Research Center, Via Alessandro Manzoni 56, 20089 \\ Rozzano, Milan, Italy. Email: pierluigi.novellis@cancercenter.humanitas.it.
}

Background: Robotic surgery is increasingly used to resect lung cancer. However costs are high. We compared costs and outcomes for robotic surgery, video-assisted thoracic surgery (VATS), and open surgery, to treat non-small cell lung cancer (NSCLC).

Methods: We retrospectively assessed 103 consecutive patients given lobectomy or segmentectomy for clinical stage I or II NSCLC. Three surgeons could choose VATS or open, the fourth could choose between all three techniques. Between-group differences were assessed by Fisher's exact, two-way analysis of variance (ANOVA), and Wilcoxon-Mann-Whitney test. $\mathrm{P}$ values $<0.05$ were considered significant.

Results: Twenty-three patients were treated by robot, 41 by VATS, and 39 by open surgery. Age, physical status, pulmonary function, comorbidities, stage, and perioperative complications did not differ between the groups. Pathological tumor size was greater in the open than VATS and robotic groups $(\mathrm{P}=0.025)$. Duration of surgery was 150, 191 and 116 minutes, by robotic, VATS and open approaches, respectively $(\mathrm{P}<0.001)$. Significantly more lymph node stations were removed $(\mathrm{P}<0.001)$, and median length of stay was shorter (4, 5 and 6 days, respectively; $\mathrm{P}<0.001$ ) in the robotic than VATS and open groups. Estimated costs were $82 \%, 68 \%$ and $69 \%$, respectively, of the regional health service reimbursement for robotic, VATS and open approaches.

Discussion: Robotic surgery for early lung cancer was associated with shorter stay and more extensive lymph node dissection than VATS and open surgery. Duration of surgery was shorter for robotic than VATS. Although the cost of robotic thoracic surgery is high, the hospital makes a profit.

Keywords: Non-small cell lung cancer (NSCLC); lobectomy open surgery; robotic surgery; thoracoscopic surgery; video-assisted surgery; costs

Submitted Jul 20, 2017. Accepted for publication Jan 20, 2018.

doi: $10.21037 /$ jtd.2018.01.123

View this article at: http://dx.doi.org/10.21037/jtd.2018.01.123 


\section{Introduction}

Early experience with da Vinci robots (Intuitive Surgical, Sunnyvale, CA, USA) to perform lung surgery, mainly for lung cancer, showed that this minimally invasive approach is feasible and safe (1-3). The technology overcomes many of the disadvantages of video-assisted thoracic surgery (VATS) in that the robot arms and attached instruments have greater precision, a superior range of motion, and improved ergonomic characteristics compared to VATS instruments, and also provide a high definition three-dimensional view of the operating field $(1,2)$.

The US Agency for Healthcare Research and Quality (4), which collects data from non-academic hospitals, found that from 2009 to 2013 robotic lobectomies increased from $1 \%$ to $11 \%$ of total lobectomies, while open lobectomies reduced from $66 \%$ to $56 \%$ of the total, and VATS lobectomies remained stable at $33 \%$. An analysis of the US Nationwide Inpatient Sample database (5) found a rapid increase in the number robotic lobectomies performed between 2008 and 2011, and also of the number of centers offering robotic lung surgery. By contrast, data from the Society of Thoracic Surgeons database (6) indicate increasing use of VATS for lobectomy in US academic centers, mainly because VATS is less costly than robotic surgery.

Most studies that addressed the issue found that robotassisted lobectomy is associated with significantly higher costs than both open surgery and VATS $(5,7-9)$ although two studies $(10,11)$ estimated lower costs for robotic than open surgery mainly due to reduced length of hospital stay. Most studies report shorter hospital stays for robotic/VATS lobectomy than open lobectomy (7-11).

As regards short-term outcomes, a 2012 systematic review (12) concluded that perioperative outcomes, including postoperative complications, appeared similar to VATS, but that robotic surgery was associated with a steep learning curve and that outcomes were likely to improve once proficiency had been reached. Higher rates of intraoperative injury and bleeding than VATS were reported in US database study (5). However, while VATS is an established procedure, in use since the early 1990s (13), robotic surgery was being introduced during the study period [2008-2011] covered by the analysis (5), so many robotic cases are likely to have been performed during the learning curve.

In fact few comparative data are available from single centers, particularly European centers, whose surgeons have attained full proficiency in robotic techniques. We therefore performed a retrospective analysis of prospectively collected data to compare clinical outcomes and costs for robotic, VATS, and open surgery in patients with early non-small cell lung cancer (NSCLC) treated at our hospital in northern Italy. Despite the focus was on cost analysis we evaluated also the perioperative and clinical outcomes of patients in different groups to assess their impact on costs like for example the different duration of surgery and the different hospital stay between groups. The surgeon who performed the robotic lobectomies had attained full proficiency in the technique prior to the period of the present study. The three other surgeons were experienced in performing both VATS and open surgery.

\section{Methods}

A consecutive series of 103 patients treated by lobectomy or segmentectomy for clinical stage I or II NSCLC from May 2015 to March 2016 was examined retrospectively. Patients with N2 disease, requiring extended surgery (chest wall, sleeves), or who had received preoperative chemotherapy or radiotherapy were excluded. Four surgeons, experienced in minimally invasive thoracic surgery, were involved: one surgeon performed robotic, VATS or open surgery according to choice although a robot was not always available; the other three surgeons performed VATS or open surgery according to choice. Operating times, adverse events, and length of hospital stay were recorded. All patients signed an informed consent at the admission in the division for the acquisition and the usage of clinical data for research purposes. For the use of data we followed the rules of the Helsinki declaration. The study was approved by the internal research board.

\section{Surgical procedure}

Open surgery is carried out using an antero-lateral muscle sparing thoracotomy at the fourth intercostal space. For VATS surgery we use a triportal anterior approach as described by Hansen and colleagues (6) with a $3 \mathrm{~cm}$ utility incision in the fourth intercostal space anterior to the latissimus dorsi muscle and two additional ports. The robotic technique consists in a four arms approach including an anterior utility incision of $3 \mathrm{~cm}$ in IV a or $\mathrm{V}$ intercostal space and no $\mathrm{CO}_{2}$ insufflation, as described in a previous paper (3). 


\section{Cost estimation}

The mean cost of each operation per group was estimated. Items considered in the estimation are shown in Table 1, which also shows mean use of each item per patient in each of three surgical groups, extracted from our database of patient records. The cost of items per patient was estimated either by applying actual costs to the amount of each item used or by making estimates: for example fixed costs (overheads and administration) were estimated at $23 \%$ of the health service reimbursement for all three approaches. Robot depreciation was estimated from the capital cost of 2 million euro, plus annual maintenance of 200,000 euro divided by number of procedures per year (400 cases) over 8 years of life. Costs were subsequently expressed as a percentage of the regional health service health reimbursement

\section{Statistical methods}

Patient- and disease-related characteristics are reported as frequencies and proportions, medians and ranges, or means and standard deviations (SD). Differences between groups for categorical data were evaluated using Fisher's exact test. For continuous data two-way analysis of variance (ANOVA) and the Wilcoxon Mann Whitney test were used. P values $<0.05$ were considered significant. The analyses were performed with STATA software V 13.

\section{Results}

As shown in Table 2, 23 patients underwent robotic surgery, 41 received VATS, and 39 received open surgery. Anatomical segmentectomy was performed in 2 robotic patients and 1 open patient. All other patients received standard lobectomy. The groups had similar age $(\mathrm{P}=0.2)$, physical status (American Society of Anesthesiologists classification) $(\mathrm{P}=0.5)$, proportions with heart $(\mathrm{P}=0.7)$ and lung $(\mathrm{P}=0.5)$ comorbidities, median proportion of vital capacity expired in 1 second (FEV1\%) $(\mathrm{P}=1.0)$, and pathological stage $(\mathrm{P}=0.25)$. Pathological tumor size was similar in the robotic and VATS groups, but higher in the open group $(\mathrm{P}=0.025)$. As shown in Table 3, duration of surgery was 150,191 , and 116 minutes in the robotic, VATS and open groups $(\mathrm{P}<0.001)$ respectively. Significantly $(\mathrm{P}<0.001)$ more lymph node stations were removed in the robotic group (mean, 4.7) than the VATS (2.7) and open (3.7) groups, and median length of stay was significantly shorter: 4,5 and 6 days for the robotic, VATS and open groups respectively $(\mathrm{P}<0.001)$. Complication rates did not differ significantly between groups $(\mathrm{P}=0.744)$.

Estimated costs (Figure 1) were $82 \%$ of Region of Lombardy lobectomy reimbursement for robotic surgery, $68 \%$ for VATS, and $69 \%$ for open surgery. Thus the hospital always made a profit, although this was fairly small for robotic surgery. The mean additional cost of robotic surgery per operation was 1,495 euro $(\$ 1,405)$ compared to VATS and 1,840 euro $(\$ 1,730)$ compared to open surgery (Table 4).

\section{Discussion}

Our findings shows that the estimated cost of robotic surgery was around $13.5 \%$ higher than for VATS and open surgery, however robotic approach was associated with a profit margin for the hospital of about $18 \%$ relative to the Region of Lombardy health service reimbursement of 11,500 euro $(\$ 12,235)$ (Figure 1). Thus, notwithstanding the fact that our robotic patients were discharged after a median of only 4 days, compared to 5 days for VATS patients, and 6 days for open patients, savings on this major item were insufficient to bring the cost of robotic surgery closer to those of VATS and open surgery. The main reimbursement equal for every kind of lobectomy independently of the type of the approach additional costs arising from robotic surgery were 2,062 euro $(\$ 1,938)$ for consumables (robot pack).

With the limitation of a small number of cases, robotic surgery was associated with shorter postoperative stay and more extensive lymph node dissection than both VATS and open surgery, as well as shorter operating time than VATS. Complications and mortality were similar between the groups.

We chose the point of view of the hospital because in Italy Public Health System Supplies and there is no additional coverage from the region for the robotic kit.

The first comparative analysis of costs was performed by Park et al. (11) in 2008. They considered 269 patients who received open lobectomy and 99 patients who underwent VATS lobectomy-with 87 having VATS alone and 12 having a robot-assisted procedure. Operating times were similar but postoperative stay was 4 days with the VATS and robotic approaches, compared to 6 days for open surgerywhich had a major effect in reducing the costs of robotic and VATS approaches compared to open surgery. In addition, the surgeon's fee was higher for open surgery-incurred for performing the thoracotomy-even though the operation 
Table 1 Average consumption of resources per patient based on actual consumption

\begin{tabular}{|c|c|c|c|c|}
\hline Cost item & Unit of measurement & $\begin{array}{l}\text { Robotic surgery } \\
\qquad(\mathrm{N}=23)\end{array}$ & $\begin{array}{l}\text { Open surgery } \\
\qquad(\mathrm{N}=38)\end{array}$ & $\begin{array}{l}\text { VATS } \\
(\mathrm{N}=42)\end{array}$ \\
\hline Days in ordinary ward & Mean days/patient & 5.00 & 8.97 & 7.50 \\
\hline Days in intensive care & Mean days/patient & 0.05 & 0.37 & 0.11 \\
\hline Operating room & Mean hours/patient & 4.01 & 3.25 & 4.29 \\
\hline Preparation time & Mean hours/patient & 1.38 & 1.16 & 1.20 \\
\hline Surgeons' time & Mean hours/patient & 10.22 & 11.40 & 12.71 \\
\hline Operating room & $\begin{array}{c}\text { Time in operating room }+30 \text { minutes } \\
\text { patient preparation }\end{array}$ & 6.72 & 5.91 & 7.96 \\
\hline Hospital stay & 30 minutes/day +1 hours clinical notes & 3.50 & 5.49 & 4.75 \\
\hline Diagnostics pre-admission & & 23 & 21 & 21 \\
\hline Lab tests & Mean/patient & 13.9 & 13.8 & 12.0 \\
\hline Consultation & Mean/patient & 6.7 & 4.5 & 6.9 \\
\hline CT & Mean/patient & 0.9 & 0.8 & 0.7 \\
\hline Spirometry & Mean/patient & 0.8 & 0.6 & 0.8 \\
\hline X-ray & Mean/patient & 0.8 & 0.7 & 0.7 \\
\hline Microbiology & Mean/patient & 1 & 2 & 1 \\
\hline Consultation & Mean/patient & 0 & 0 & 1 \\
\hline Bronchoscopy & Mean/patient & 0 & 1 & 1 \\
\hline Other & Mean/patient & 0 & 1 & 0 \\
\hline Diagnostics after discharge & & 1 & 0 & 0 \\
\hline Consultation & Mean/patient & 0 & 0 & 0 \\
\hline Pathology & Mean/patient & 0 & 0 & 0 \\
\hline Lab tests & Mean/patient & 1 & 0 & 0 \\
\hline X-ray & Mean/patient & 0 & 0 & 0 \\
\hline
\end{tabular}

Table 1 (continued) 
Table 1 (continued)

\begin{tabular}{|c|c|c|c|c|}
\hline Cost item & Unit of measurement & $\begin{array}{l}\text { Robotic surgery } \\
\qquad(\mathrm{N}=23)\end{array}$ & $\begin{array}{l}\text { Open surgery } \\
\qquad(\mathrm{N}=38)\end{array}$ & $\begin{array}{l}\text { VATS } \\
(\mathrm{N}=42)\end{array}$ \\
\hline Medications & & 31 & 99 & 62 \\
\hline Antiplatelet, antipyretics, analgesics, NSAIDs & Mean administrations/patient & 5 & 17 & 12 \\
\hline Drips/ parenteral nutrition & Mean administrations/patient & 3 & 7 & 6 \\
\hline Antibiotics & Mean administrations/patient & 3 & 7 & 5 \\
\hline Other & Mean administrations/patient & 10 & 27 & 14 \\
\hline Scalpels, filament, needles & Mean/operation & 6 & 13 & 7 \\
\hline Robot pack & Mean/operation & 1 & 0 & 0 \\
\hline Blood products & Packs/patient & 0.00 & 0.11 & 0.20 \\
\hline
\end{tabular}

NSAIDs, nonsteroidal anti-inflammatory drugs.

Table 2 Characteristics of 103 lung cancer patients and their disease according to surgical approach (robotic, VATS, open)

\begin{tabular}{|c|c|c|c|c|}
\hline Analyzed variables & Robotic surgery $(\mathrm{N}=23)$ & VATS $(\mathrm{N}=42)$ & Open surgery ( $\mathrm{N}=38)$ & $P$ value \\
\hline ASA physical status classification & & & & 0.514 \\
\hline $1-2, \mathrm{n}(\%)$ & $19(86.36)$ & $32(80.00)$ & $28(73.68)$ & \\
\hline $3, \mathrm{n}(\%)$ & $3(13.64)$ & $8(20.00)$ & $10(26.32)$ & \\
\hline \multicolumn{5}{|l|}{ Comorbidities } \\
\hline Cardiac, n (\%) & $2(8.70)$ & $3(7.14)$ & $5(13.16)$ & 0.700 \\
\hline Pulmonary, n (\%) & $3(13.04)$ & $2(4.76)$ & $3(7.89)$ & 0.454 \\
\hline Pathologic stage & & & & 0.252 \\
\hline Pathologic tumor size, cm, median (range) & $2.1(0.8-11.5)$ & $2.2(0.5-7.0)$ & $3(0.7-7.2)$ & 0.025 \\
\hline
\end{tabular}

VATS, video-assisted thoracic surgery; ASA, American Society of Anesthesiologists; FEV1\%, proportion of vital capacity expired in 1 second. 
Table 3 Perioperative outcomes in robotic, VATS and open surgery patients

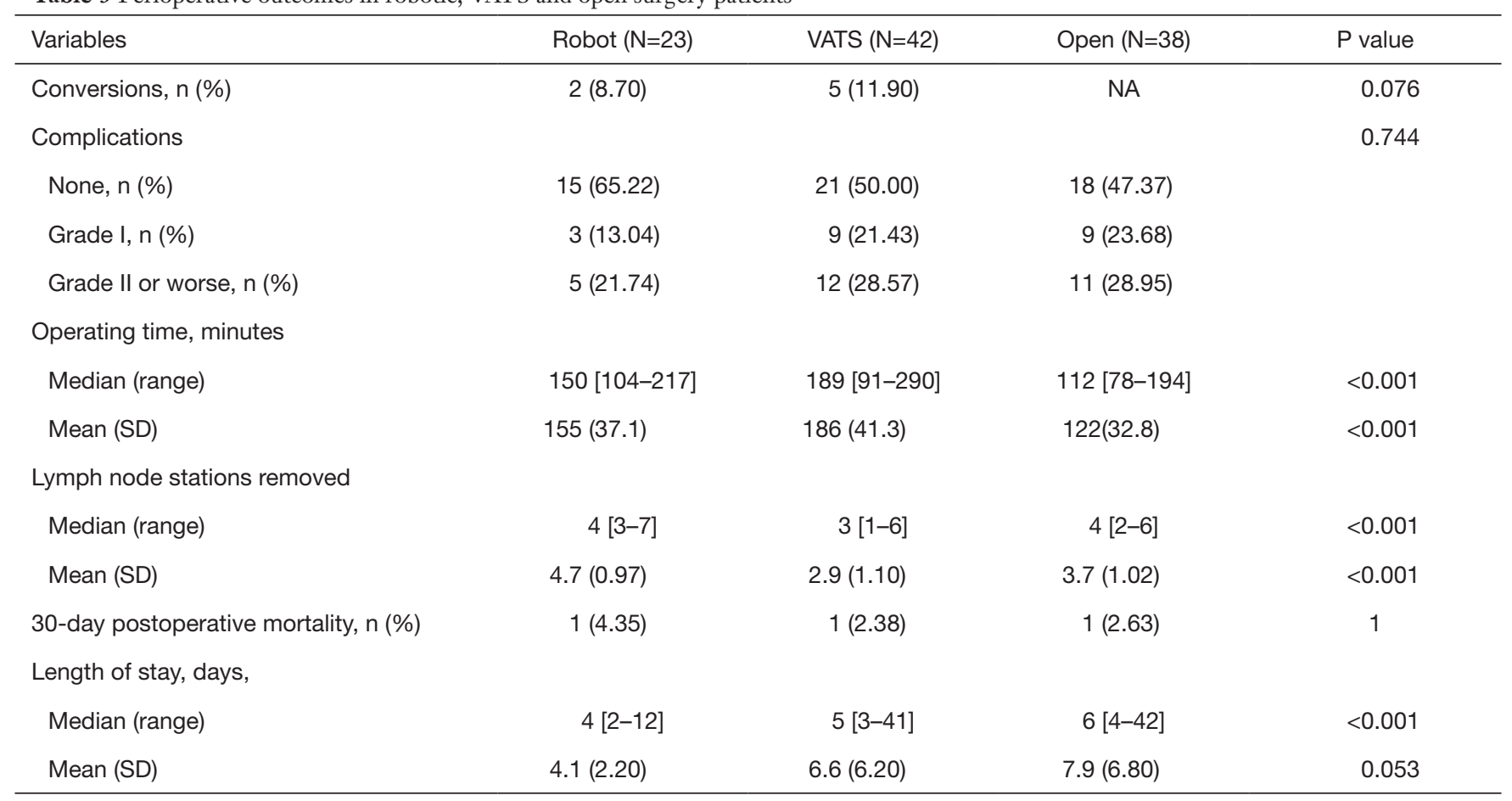

NA, not applicable; VATS, video-assisted thoracic surgery; SD, standard deviations.

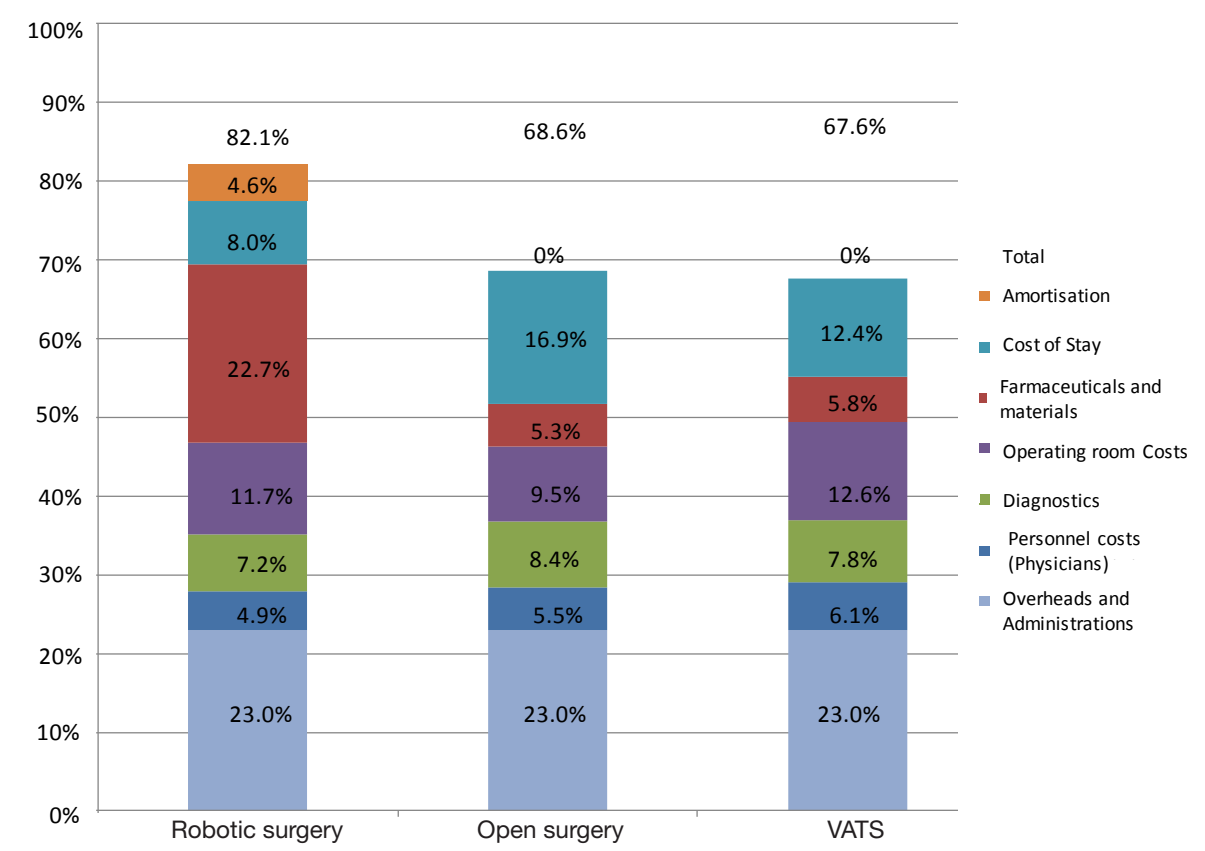

Figure 1 The estimated cost of robotic surgery was higher than for VATS and open surgery, with a profit margin of about $18 \%$ relative to the Region of Lombardy health service reimbursement of 11,500 euro (\$12,235). Estimated costs were 82\% of Region of Lombardy lobectomy reimbursement for robotic surgery, $69 \%$ for open surgery, and $68 \%$ for VATS. VATS, video-assisted thoracic surgery. 
Table 4 Summary of mean direct costs per patient in euro from the data shows in Table 1

\begin{tabular}{lccc}
\hline Variables & Robot & VATS & Open \\
\hline Personnel & 562 & 699 & 627 \\
$\begin{array}{l}\text { Medications, instruments } \\
\text { and accessories }\end{array}$ & 545 & 669 & 615 \\
Robot consumables & 2,062 & 0 & 0 \\
Diagnostics & 829 & 894 & 964 \\
Operating room costs & 1,349 & 1,443 & 1,094 \\
Hospital stay & 920 & 1,427 & 1,944 \\
Depreciation & 532 & 0 & 0 \\
Total & 6,799 & 5,132 & 5,244 \\
\hline
\end{tabular}

VATS, video-assisted thoracic surgery.

(anatomic lobectomy) was identical by each approach. Robotic surgery cost $\$ 3,981$ more than VATS but $\$ 3,988$ less than open surgery. The robot excess over VATS was mainly due to the costs of robotic disposables and drapes. Although this analysis (11) did not consider depreciation, the authors cited another study which estimated an additional $\$ 857$ per robotic case due to amortization of the robot instrument. Even considering depreciation, robotic surgery was still cheaper than open surgery. These findings contrast with ours in that we estimated medical personnel costs to be highest for VATS and lowest for robotic, with only a 137 euro (\$129) difference between the two. Like Park et al. (11), we found that robot amortization contributed considerably to robotic costs ( $4.6 \%$ of total) against $0 \%$ for open surgery and VATS.

A more recent single institute study from Deen et al. (8) on 184 consecutive patients with similar comorbidities, who underwent lobectomy or segmentectomy (69 by thoracotomy, 57 by robot, and 58 by VATS), found that overall costs, which included depreciation, differed significantly between the groups. VATS was the least expensive, and robotic surgery was the most expensive procedure. On average robotic surgery cost $\$ 3,182$ more than VATS $(\mathrm{P}<0.001)$. Although inpatient stay was shortest for robotic patients, the differences between the groups were not significant, and the higher cost of robotic surgery was mainly due to robotic-specific supplies and depreciation. The authors commented that operating times and robot consumables needed to reduce in order for robotic surgery to be competitive.

In their US database study, Paul et al. (5) estimated costs based on hospital-specific cost-to charge-ratios. The median cost of robotic lobectomies was significantly higher more than for VATS procedures $(\$ 22,582 v s . \$ 17,874, \mathrm{P}<0.001)$. Swanson et al. (9) analyzed costs in propensity scorematched patients from a large US multihospital database, again finding that robotic lobectomies were more expensive than VATS lobectomies $(\$ 21,833$ vs. $\$ 18,080)$. Neither study $(5,7)$ identified sub-items mainly responsible for the greater cost of robotic surgery or considered depreciation.

In their 2014 study Nasir et al. (10) assessed the costs and profitability for 394 planned robotic anatomic resections (282 lobectomies, 71 segmentectomies, and 41 conversions to open surgery) carried out by a single surgeon. Median total expenses were estimated at $\$ 15,440$ per patient against a median Medicare reimbursement of $\$ 18,937$ per patient, so that the median profit per patient was $\$ 3,497$. These figures, which do not consider non-Medicare patients, are similar to ours.

Turning now to outcomes, we note two recent US database studies $(5,9)$ concluded that robotic surgery offered no advantages over VATS for lung cancer surgery. Paul et al. (5), who compared perioperative outcomes in patients given robot-assisted lobectomy with those given thoracoscopic lobectomy in 2008-2011 (2,478 robotic lobectomies; 37,595 VATS lobectomies) found that robotic patients had significantly more cardiovascular complications and iatrogenic bleeding complications. However, this study had the limitation that $40 \%$ of robotic surgeries were performed in small-to-medium sized hospitals; similar proportions were non-teaching and had a moderate patient volume. Swanson et al. (9) found similar complications and length of hospital stay for robotic and VATS lobectomies, however they were aware of a possible bias because only a few of the hospitals investigated were performing robotic procedures. They therefore compared a single hospital which had performed 126 robotic lobectomies with a single non-robotic hospital with otherwise similar characteristics. They again found similar outcomes for both approaches, but uncovered a significantly greater proportion of minor adverse events in patients who received a robotic lobectomy.

The study of Deen et al. (8), which assessed 184 consecutive operations, found no differences in complication rates or length of hospital stay, but times in the operating room were significantly longer in the robotic (223 minutes) than the VATS (202 minutes) or open (180 minutes) groups. In our study operating times were generally shorter, with a median of only 150 minutes for the robotic approach (Table 3), indicating that a proficient surgeon can complete a robotic 
lobectomy in a relatively short time. This is supported by the largest single-surgeon experience so far (10) in which median operating time for lobectomy/segmentectomy was 107 minutes (range, 48-399 minutes) but included $10 \%$ of cases converted to open surgery. Although this was not a comparative study, the authors (10) concluded that robot-assisted lobectomy for cancer offered excellent lymph-node removal, and minimal morbidity and pain. Our data are consistent with these conclusions in that the robotic approach afforded superior lymph node dissection compared to both open surgery and VATS.

We note finally the encouraging long-term findings of a 2016 retrospective analysis (14). This study analyzed 2,132 patients treated by lobectomy for clinical stage I NSCLC, with choice of approach left to the surgeon (184 robotic, 761 VATS, 1,187 open). Similar long-term survival was found in propensity score-matched groups, although length of stay was shorter in the robotic and VATS groups, and more lymph nodes were removed in the robotic group.

The main limitations of the present study are its retrospective nature and possible selection bias. Pathological tumor size was significantly larger in the open group suggesting that surgeons tended to select the open approach for more challenging cases. However precise selection criteria were used: consecutive patients treated over a short period at a single institute by standard lobectomy or segmentectomy for clinical stage I-II NSCLC. Another limitation was the fact that only one surgeon performed robotic surgery, while the other three were proficient in VATS and open surgery. Study strengths are that we analyzed the experience of four surgeons who had gained full proficiency in the minimally invasive techniques they employed. To our knowledge this is the first study from a European hospital to produce a comparative cost analysis for lung cancer patients.

To conclude, the high current cost of robotic thoracic surgery is worrying and may limit its uptake by thoracic surgeons in Europe. However the approach was profitable for our hospital in that it cost about $18 \%$ less than the current health service reimbursement. In addition, the significantly shorter length of hospital stay for robotic patients is useful for hospital management as it frees beds for other patients helping to optimize resources and shorten waiting times.

\section{Acknowledgements}

The authors thank Don Ward for help with the English, and acknowledge support from the Umberto Veronesi Foundation for a fellowship to P Novellis and E Dieci.

\section{Footnote}

Conflicts of Interest: G Veronesi is a consultant for ABI Medica SpA and Medtronic, other authors have no conflicts of interest to declare.

Ethical Statement: All patients signed an informed consent at the admission in the division for the acquisition and the usage of clinical data for research purposes. For the use of data we followed the rules of the Helsinki declaration. The study was approved by the internal research board.

\section{References}

1. Melfi FM, Menconi GF, Mariani AM, et al. Early experience with robotic technology for thoracoscopic surgery. Eur J Cardiothorac Surg 2002;21:864-8.

2. Park BJ, Flores RM, Rusch VW. Robotic assistance for video-assisted thoracic surgical lobectomy: technique and initial results. J Thorac Cardiovasc Surg 2006;131:54-9.

3. Veronesi G, Galetta D, Maisonneuve P, et al. Four-arm robotic lobectomy for the treatment of early-stage lung cancer. J Thorac Cardiovasc Surg 2010;140:19-25.

4. Free Health Care Statistics. Available online: https:// hcupnet.ahrq.gov/\#setup.

5. Paul S, Jalbert J, Isaacs AJ, et al. Comparative effectiveness of robotic-assisted vs thoracoscopic lobectomy. Chest 2014;146:1505-12.

6. Hansen HJ, Petersen RH, Christensen M. Videoassisted thoracoscopic surgery (VATS) lobectomy using a standardized anterior approach. Surg Endosc 2011;25:1263-9.

7. Louie BE, Wilson JL, Kim S, et al. Comparison of VideoAssisted Thoracoscopic Surgery and Robotic Approaches for Clinical Stage I and Stage II Non-Small Cell Lung Cancer Using The Society of Thoracic Surgeons Database. Ann Thorac Surg. 2016;102:917-24.

8. Deen SA, Wilson JL, Wilshire CL, et al. Defining the cost of care for lobectomy and segmentectomy: a comparison of open, video-assisted thoracoscopic, and robotic approaches. Ann Thorac Surg 2014;97:1000-7.

9. Swanson SJ, Miller DL, McKenna RJ Jr, et al. Comparing robot-assisted thoracic surgical lobectomy with conventional video-assisted thoracic surgical lobectomy and wedge resection: results from a multihospital database 
(Premier). J Thorac Cardiovasc Surg 2014;147:929-37.

10. Nasir BS, Bryant AS, Minnich DJ, et al. Performing robotic lobectomy and segmentectomy: cost, profitability, and outcomes. Ann Thorac Surg 2014;98:203-8.

11. Park BJ, Flores RM. Cost comparison of robotic, videoassisted thoracic surgery and thoracotomy approaches to pulmonary lobectomy. Thorac Surg Clin 2008;18:297-300.

12. Cao C, Manganas C, Ang SC, et al. A systematic review and meta-analysis on pulmonary resections by robotic video-assisted thoracic surgery. Ann Cardiothorac Surg

Cite this article as: Novellis $\mathrm{P}$, Bottoni E, Voulaz E, Cariboni U, Testori A, Bertolaccini L, Giordano L, Dieci E, Granato L, Vanni E, Montorsi M, Alloisio M, Veronesi G. Robotic surgery, video-assisted thoracic surgery, and open surgery for early stage lung cancer: comparison of costs and outcomes at a single institute. J Thorac Dis 2018;10(2):790-798. doi: 10.21037/ jtd.2018.01.123
2012;1:3-10.

13. Roviaro GC, Rebuffat C, Varoli F, et al. Major videothoracoscopic pulmonary resections. Endosc Surg Allied Technol 1993;1:288-93.

14. Yang HX, Woo KM, Sima CS, et al. Long-term Survival Based on the Surgical Approach to Lobectomy For Clinical Stage I Nonsmall Cell Lung Cancer: Comparison of Robotic, Video-assisted Thoracic Surgery, and Thoracotomy Lobectomy. Ann Surg 2017;265:431-7. 